\title{
Research Paper \\ Optimization of PET Expression Vector for Fusion of Recombinant Protein and Elastin-Like Polypeptide Biopolymer
}

\author{
Mohammad Reza Soleyman $^{1} \odot$, Mostafa Khalili ${ }^{2,3}$, , Ali Reza Soleiman Meiguni ${ }^{4} \odot{ }^{*}$ Maryam Baazm $^{5,6}$
}

1. Department of Biotechnology, School of Medicine, Arak University of Medical Sciences, Arak, Iran.

2. Blood Transfusion Research Center, High Institute for Research and Education in Transfusion Medicine, Tehran, Iran.

3. Blood Transfusion Center, Arak, Iran.

4. Department of Management, Yadegar Emam Khomeini Branch, Islamic Azad University, Shahre Rey, Iran.

5. Department of Anatomy, School of Medicine, Arak University of Medical Sciences, Arak, Iran.

6. Cellular and Molecular Research Center, Arak University of Medical Sciences, Arak, Iran.

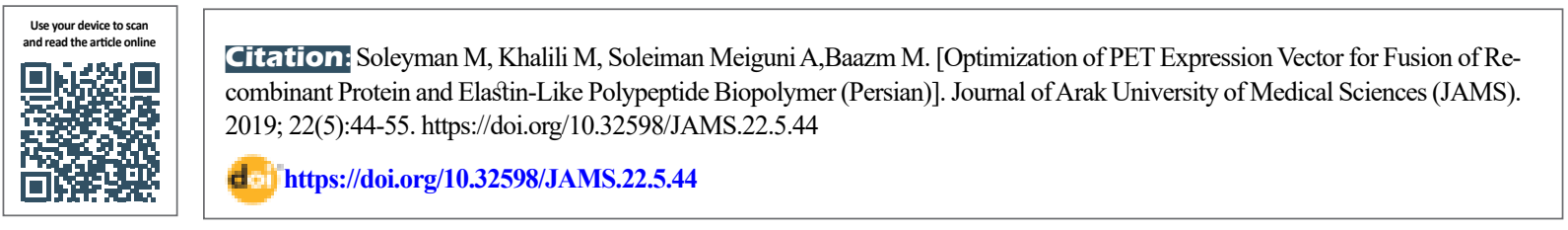

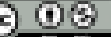

Article Info:

Received: 18 May 2019

Accepted: 27 Aug 2019

Available Online: $01 \operatorname{Dec} 2019$

Key words:

Elastin-Like

Polypeptide,

Recombinant protein

Protein fusion

\section{ABSTRACT}

Background and Aim recombinant DNA technique is a powerful and appropriate method for the production of protein biopolymers with specificity in amino acid sequence and spatial chemistry. Elastin-Like Polypeptide (ELP) is a biocompatible, biodegradable and non-immunological biopolymer used in various biotechnology studies. The ELP tag is a cheap, fast and non-chromatographic technique for purifying target proteins. In this study, pET expression vector was designed for the combination of ELP gene sequences and target recombinant protein in order to produce recombinant fusion protein with the ELP tag. Methods \& Materials MOD gene was transformed to E. coli-BL21 (DE3) cells after designing and synthesis among the Xbal and Xhol restriction sites in the pET-32a (+) vector of the clone. Then, colonies were isolated based on plasmid size and examined by cutting using restriction enzymes. The final recombinant colonies was verified using polymerase chain reaction method and DNA sequencing.

Ethical Considerations The Research Ethics Committee of Arak University of Medical Sciences approved all ethical considerations ofworking on laboratory animals (Code: 92-146-11).

Results Replacing the MOD sequence in the pET-32a vector (+) eliminated the components expressing the fusion tags (Thioredoxin, Histidine, and S-tag), the identification site of protease enzyme (tobacco etch virus), and multiple cloning site. In addition, it added specific restriction enzyme identification sequences of ELP gene and target gene. As a result, in the optimized pET-MODvector, 466 nucleotides reduced in size and the secondary structure was improved.

Conclusion Considering the improvement of spatial structure and reduction of pET-MOD vector size, as well as the possibility of the fusion of recombinant protein with the ELP tag, it is possible to use this vector for ELPyation of the target protein.

\section{Extended Abstract}

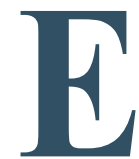

\section{Introduction}

lastin-Like Polypeptides (ELPs) are a group of temperature-sensitive biopoly- mers with various biotechnological and biomedical applications [6]. ELP is a component of temperature-sensitive synthetic biopolymers, with its solubility dependent on temperature change; under the Transition temperature $(\mathrm{Tt})$, ELP is soluble in aqueous medium and accumulates in the form of self-assembly with increased solution temperature.

\section{* Corresponding Author:}

Maryam Baazm, PhD.

Address: Department of Anatomy, School of Medicine, Arak University of Medical Sciences, Arak, Iran.

Tel: +98 (916) 6621131

E-mail: dr.baazm@arakmu.ac.ir 
By binding the protein at the gene level to the ELP, the resulting aggregated proteins will have the same properties [7]. Due to the thermal sensitivity of the ELP molecule, purification by chromatography is not required for protein purification [5]. Studies to produce the fusion of recombinant protein bound to the ELP tag, as well as the oligomerization of ELP, require several cloning steps in the nonspecific vector and re-subcloning $[13,15]$. Therefore, in this study, we optimized the pET expression vector, the pET-MOD specific vector, to oligomerize ELP, as well as to produce recombinant ELP tag fusion protein for biotechnological and pharmaceutical applications. Accordingly, the inexpensive purification of the recombinant protein by the Inverse Transition Cycling (ITC) technique and the targeted drug conjugate delivery, etc. will be generated.

\section{Materials and Methods}

The MOD gene was designed with the structure required for the cloning, expression, and purification of the recombinant protein. Then, the $\mathrm{XbaI}$ and $\mathrm{XhoI}$ restriction enzyme cleavage sites were added at the 5 'and 3' ends, respectively. The prediction of secondary structures and stability of mRNA expressed in pET-32a $(+)$ and pET-MOD were evaluated by the centerfold server. Additionally, the $\mathrm{G}+\mathrm{C}$ content of these two fragments was evaluated by Rare Codon Analysis Tools. The designed gene was synthesized by the Biomatic Spa company. The designed pUC-57 gene carrier was cleaved by XbaI and XhoI enzymes to release the synthetic MOD fragment.

The pET-32a(+) vector was also cleaved by $\mathrm{XbaI}$ and XhoI enzymes and dephosphorylated by alkaline phosphatase to linearize the plasmid. The purification of linearized plasmid and MOD cut DNA was performed by agarose gel purification kit. The $\mathrm{pET}$ vector and the MOD extension fragment were joined by T4 DNA ligase to form the pETMOD vector. The obtained vectors were heat-transfected to $100 \mu \mathrm{L}$ of E.coli-BL21 (DE3) cells transfected using $\mathrm{CaCl}$; then, the bacteria were spread on a cell culture plate containing Luria-Bertani medium and $100 \mu \mathrm{g} / \mathrm{mL}$ ampicillin. They were incubated overnight at $37^{\circ} \mathrm{C}$. After transformation, recombinant colonies were separated based on plasmid size. Furthermore, the positive colonies were examined for the presence of insert fragment using restriction enzyme cleavage analysis. The PCR colonization technique conducted the final confirmation of recombinant colonies, and the PCR product was confirmed by $1 \%$ agarose gel and DNA sequencing.

\section{Results}

Predicting mRNA secondary structure suggested that the optimization of the $\mathrm{pET}$ expression vector could prevent the formation of a stable secondary structure. The free energy of the second mRNA structure also changed from -122.8 to -20 . Besides, the percentage of the $\mathrm{G}+\mathrm{C}$ content of these two genes was similar and in the ideal range (50\%-51\%). After the enzymatic digestion of pUC-57, the $111 \mathrm{bp}$ fragment was released from the MOD synthesized gene; then, it was ligated into the $\mathrm{XbaI}$ and XhoI sequences and replaced with the $577 \mathrm{bp}$ sequence from the pET-32a(+) sequence.

In this optimized nucleotide sequence, genes expressing amino acid sequences, including Trx-Tag with His-Tag (thrombin protease enzyme digestion site), S-tag (TEV protease enzyme digestion site), and multiple cloning site (multiple cloning site) followed, were deleted. The pET$32 \mathrm{a}(+)$ vector length decreased from5900 bp to $5434 \mathrm{bp}$ (466bp decrease). Furthermore, the identification site of the restriction enzymes Sfi-I, BamH-I, and EcoR-I were replaced with the pET-MOD sequence. By the cleavage of the BamH-I and EcoR-I sequences, adherent sequences were created to bind the target protein gene with a similar tail. After the cleavage of the pET-MOD vector with SfiI, a linear plasmid with adherent ends consistent with the adherent end of the ELP gene cut by Bgl-I and pflM-I was generated. The nucleotide sequence encoding GGSGGSG (glycine + cysteine) was added to the MOD sequence, as a flexible linker region between the target protein sequence and the ELP tag.

Moreover, the WYWYW (tryptophan + tyrosine) coding nucleotide sequence was added to the MOD sequence to estimate recombinant protein concentration. The recombinant plasmid was purified from the transformed cell and identified based on plasmid size. Eventually, colony-PCR was performed to confirm the recombinant $\mathrm{pET}-\mathrm{MOD}$ plasmid. Besides, the presence of $160 \mathrm{bp}$ band on 1\% agarose gel and the sequencing of the resulting gene revealed the accuracy of cloning. The resulting pET-MOD plasmid sequence is available (Code: KP834588.1).

\section{Discussion}

The production of large amounts of the bioactive fusion protein is a critical issue in biotechnology, and ELP fusion is an appropriate choice for this purpose [21]. The ELP tag, like many other tags, might reduce the bioactivity of the fusion protein based on the size and orientation of it [23]. Studies on the production of recombinant protein fusion and the ELP tag, have used the full-length synthesis of the target gene to put these 
genes together. As a result, that method will be costly, and the sequence development will be complex and time-consuming (due to its timely nature) [24].

Alternatively, primary cloning in the non-expression vector, followed by sub-cloning, was applied. This method is also time-consuming, and because of the non-specificity of vectors, oligomerization will be complicated $[25,26]$. The present study, for the first time, used the pET vectors and the MOD synthetic gene, to design and generate the pETMOD specific vector in high volumes of the bioactive fusion protein with the oligomerization ability of the ELP tag. The synthetic MOD gene was designed based on the following requirements: 1 . Reduce the sequence length of the pET-MOD vector by omitting the unnecessary sequence in the pET-32a $(+)$ base vector; 2 . Add Sfi-I locus sequence sequences that complement the inserted gene resulting from the oligomerization of the ELP gene generated by the RDL technique. Furthermore, it will reduce the complexity of the oligomerization of the ELP gene in non-specific vectors; 3. Incorporate the sequence identification of BamHI and EcoRI to the sequence integration and construction of fusion protein with ELP; 4. Incorporate a nucleotide sequence box encoding a flexible linker between the ELP tag and the target protein. This process helps to reduce the spatial interference on recombinant protein activity, particularly concerning its effect on the function of ELP5 tag fusion growth factors. The incorporation of the nucleotide sequence box encoding aromatic side-chain amino acids to absorb the UV light of the recombinant protein (to calculate protein concentration), particularly for proteins lacking aromatic amino acids [29].

\section{Conclusion}

The pET-MOD properties highlight the appropriate application of this vector for purifying recombinant protein and generating fusion protein with ELP tag (for use as a scaffold containing elastin as part of extracellular matrix). Furthermore, considering the presence of growth factors based on the study purpose, it is recommended for various studies, including wound healing research.

\section{Ethical Considerations}

\section{Compliance with ethical guidelines}

This study obtained its ethical approval form the Research Ethics Committee of Arak University of Medical Sciences (code: 92-146-11).

\section{Funding}

This study received financial support from the Deputy for Research and Technology of this university.

\section{Authors' contributions}

Investigation and initial draft preparation: Mohammad Reza Soleyman and Mostafa Khalili; Initial draft preparation and data analysis: Ali Reza Soleiman Meiguni; Review \& editing, supervision, project administration: Maryam Baazm.

\section{Conflicts of interest}

The authors declare no conflict of interest.

\section{Acknowledgements}

The authors would like to thank the Deputy for Research and Technology of Arak University of Medical Sciences for their support. 


\title{
بهينهسازى وكتور بيانى PET جهت فيوثن كردن بروتئين نوتركيب و بيويليمر يلىيبيد شبهالاستين
}

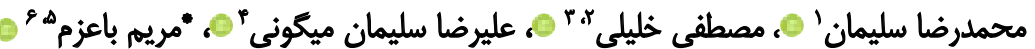 \\ ا. ا. كروه بيوتكنولورثى و ميكروبيولورىى، دانشكده يزشكى، دانشكاه علوم يزشكى اراك، اراك، ايران.

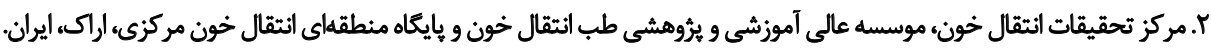

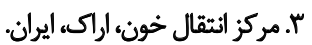

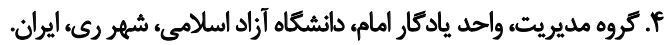

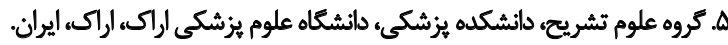

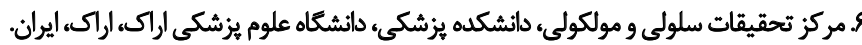

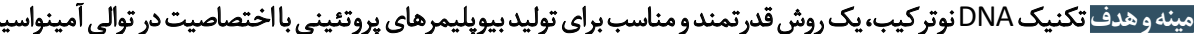

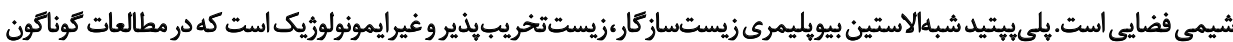

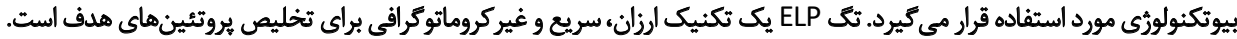

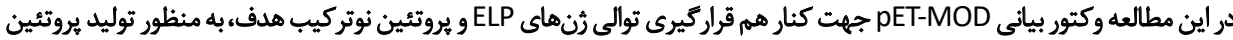

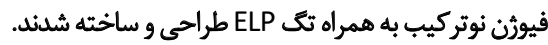

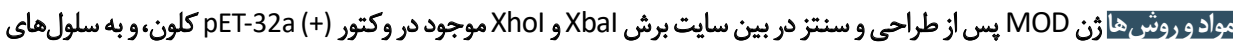

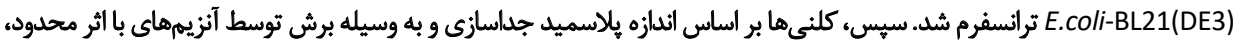

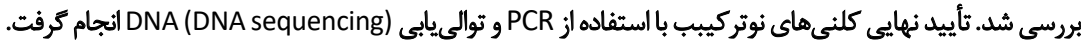

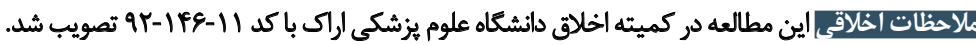

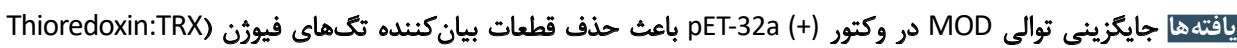
Histidine:HIS، S-tag

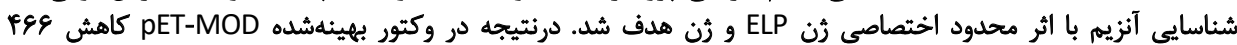

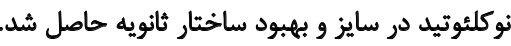

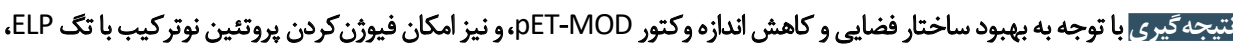

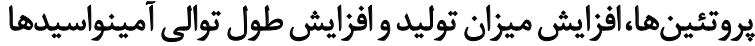

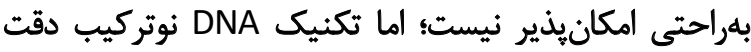

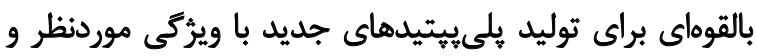

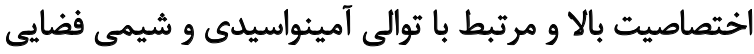

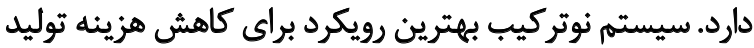

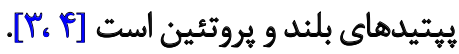

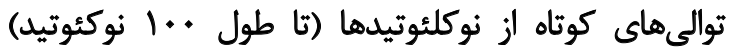
هىتواند توسط سنتز شيميايى توليد و سيس براي كدكردن
بيويليمرهاى طبيعى و مصنوعى دستهاى از مواد هستند كه

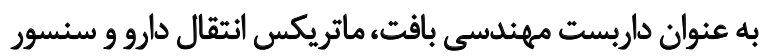

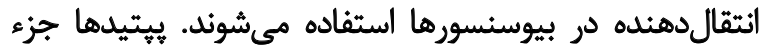

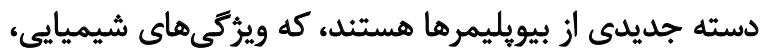

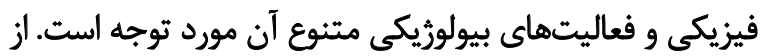

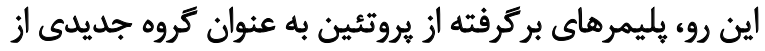

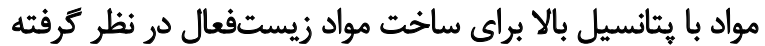

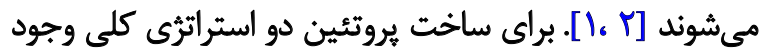
نشانى: اراك، دانشكاه علوم يزشكى اراك، دائشكده يزشكى، كروه علوم تشريح.

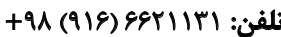
يست الكترونيكى: dr.baazm@arakmu.ac.ir 
ITC

\section{مواد وروش رها}

در اين مطالعه بنيادى كاربردى، ميزبان E.coli سويه (t) DH5a (Invitrogen)

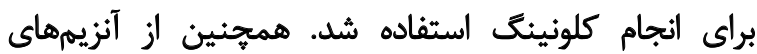

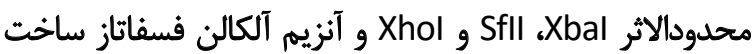

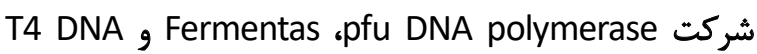
Ligase ساخت شركت Vivantis در اين تحقيق استفاده شد.

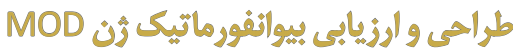

ثن MOD شامل توالى هاي نوكلئوتيدى شاين دلكارنو هبه عنوان

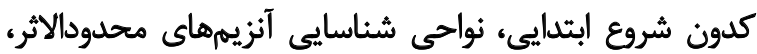

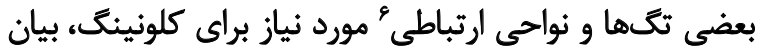

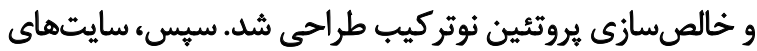

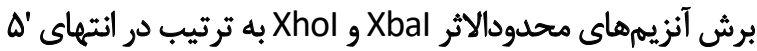

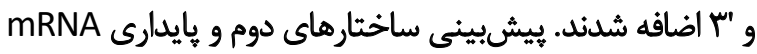
بيانشونده در (+) pET-32a

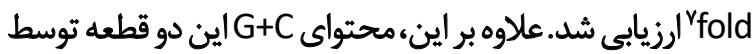

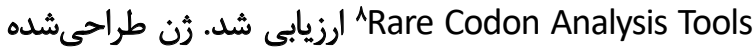

توسط شركت بيوماتيك (Biomatik, Canada) سنتز شد.

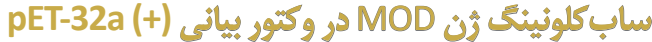

اصول مهندسى رُنتيك بر اساس اصول سمبروك و همكاران به

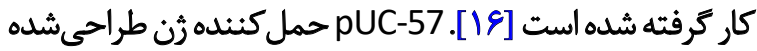
سنتزى MOD توسط شركت بيوماتيك (Biomatik, Canada)،

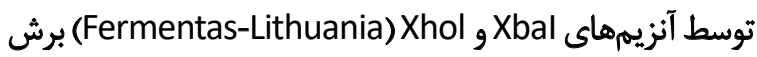

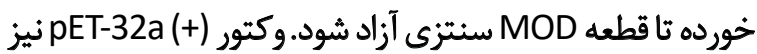

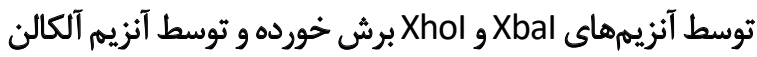

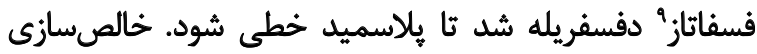

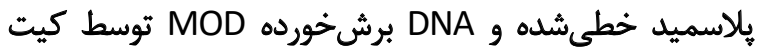

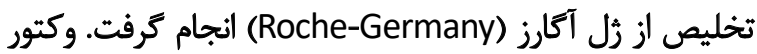

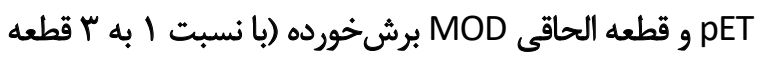

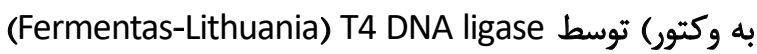
به هم ملحق شدند تا وكتور pET-MOD توسط ماصل شود. وكتورهاى حاصل از طريق روش شوك حرارتى (9 ب درجه

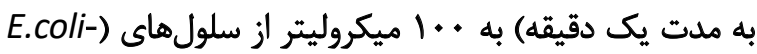

\footnotetext{
4. Inverse Transition Cycling (ICT)

5. Shine-Dalgarno

6. Linker

7. Http://www.ncrna.org/centroidfold

8. Http://www.genscript.com

9. Fermentas-Lithuania
}

يلىيتيدهاي تكرارى با وزن مولكولى موردنظر به صورت

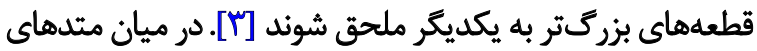

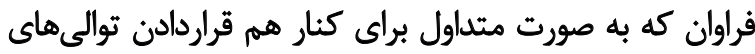

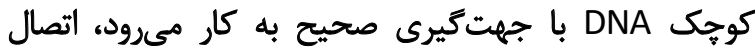

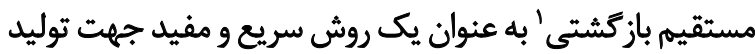

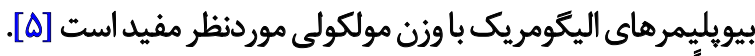

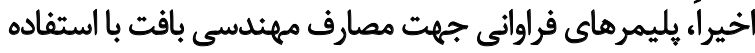

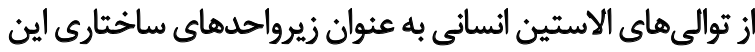

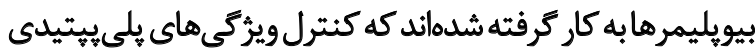

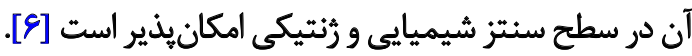

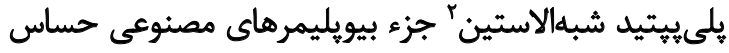

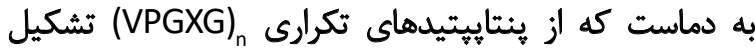

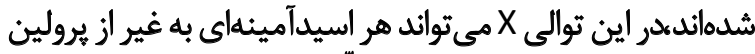

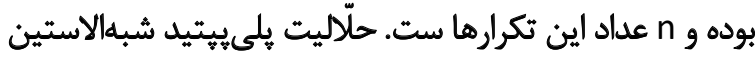

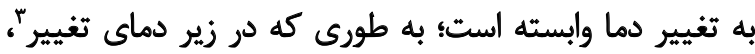

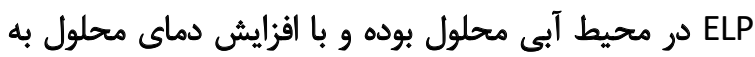

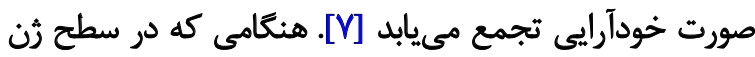

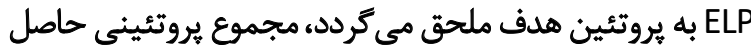

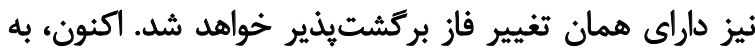

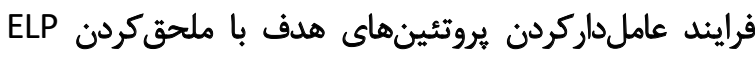

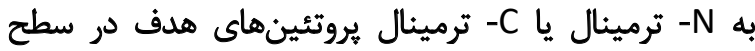

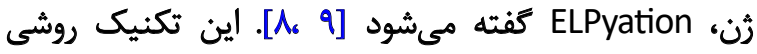

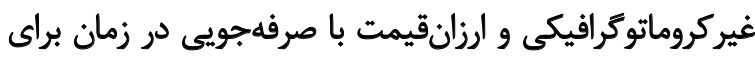

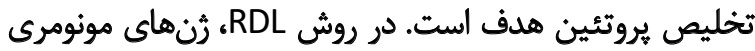

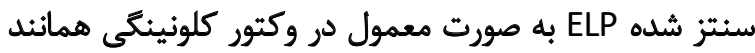

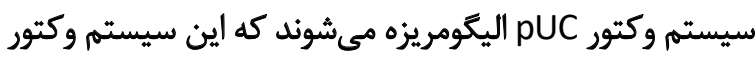

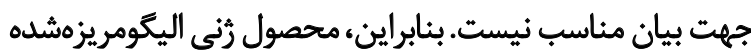

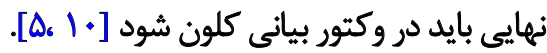
در مطالعات مختلفى به منظور تخليص غيرگروماتوكرافى و وني

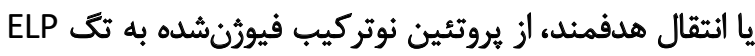

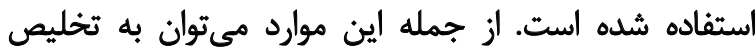

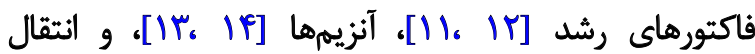

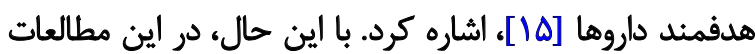

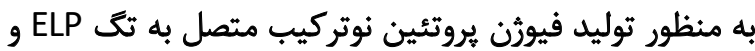

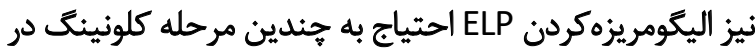

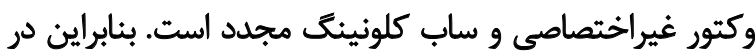

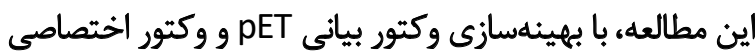

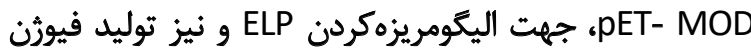

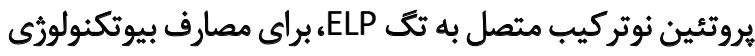

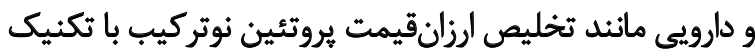

1. Recursive Directional Ligation (RDL)

2. Elastin Like Polypeptide (ELP)

3. Transition Temperature (TT) 


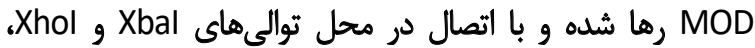

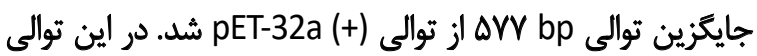

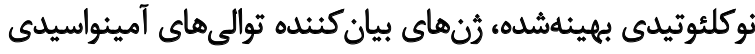

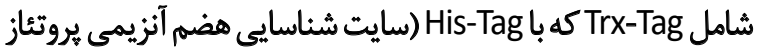

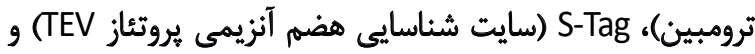

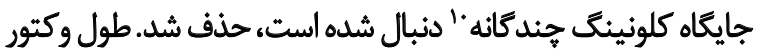

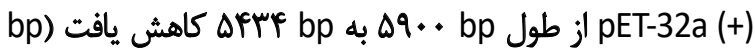

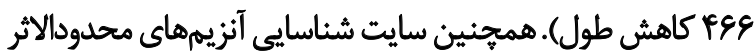

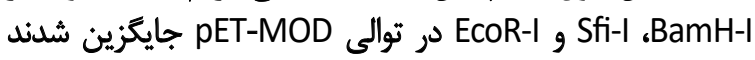

$$
\text { (تصوير شماره ب). }
$$

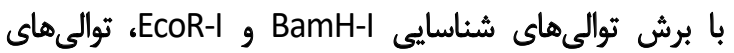

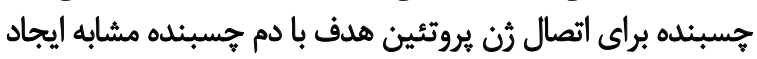

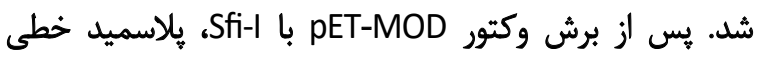

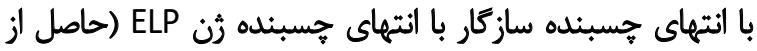

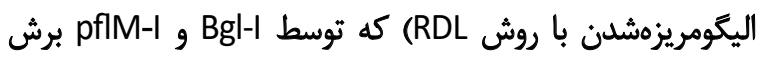

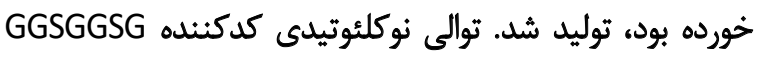

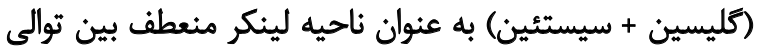

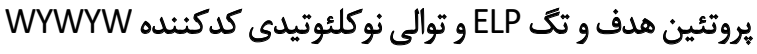

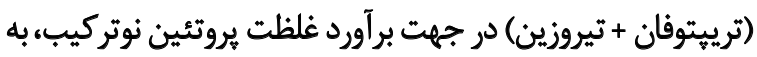
توالى MOD اضافه شد (تصوير شماره f).

تيلاسميد نوتركيبشده از سلول ترانسفرمشده تخليص شد و

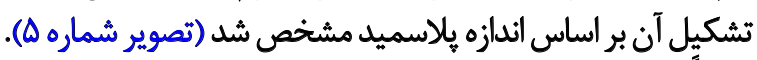

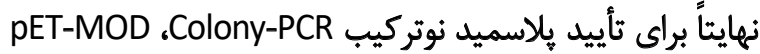

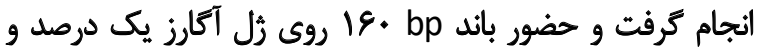
توالىيابى ثن حاصل، صحت كلونينَ ران انشان داد (تصوير شماره

10. Multiple cloning site
BL21 (DE3

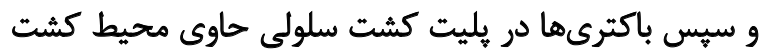
Luria-Bertani

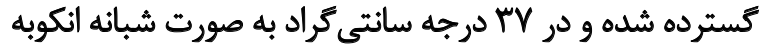

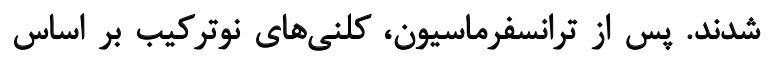

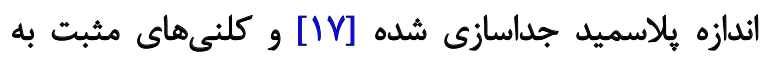

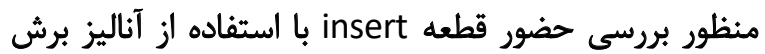

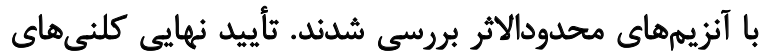

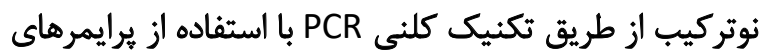
5'-GGGAATTGT- ， 5'TCAGTGGTGGTGGTGGTG-3' كه براي اين مطالعه طراحى شده بود

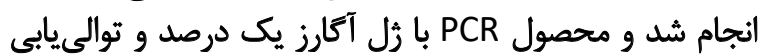
(DNA sequencing) DNA

يافتهانها

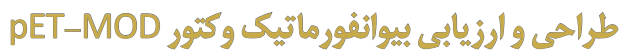
يبشبينى ساختار دوم mRNA نشان داد بهينهسازى وكتور بيانى

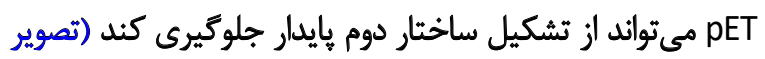

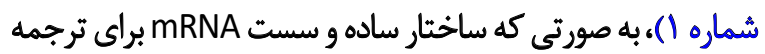

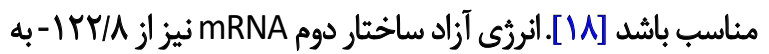

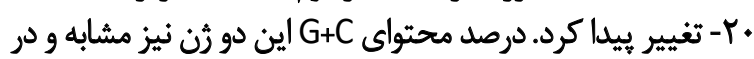

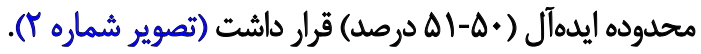

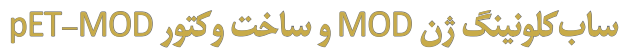

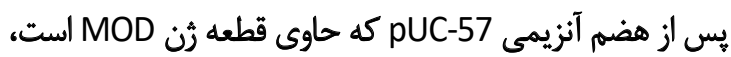

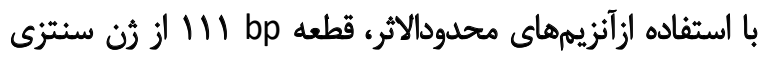

A

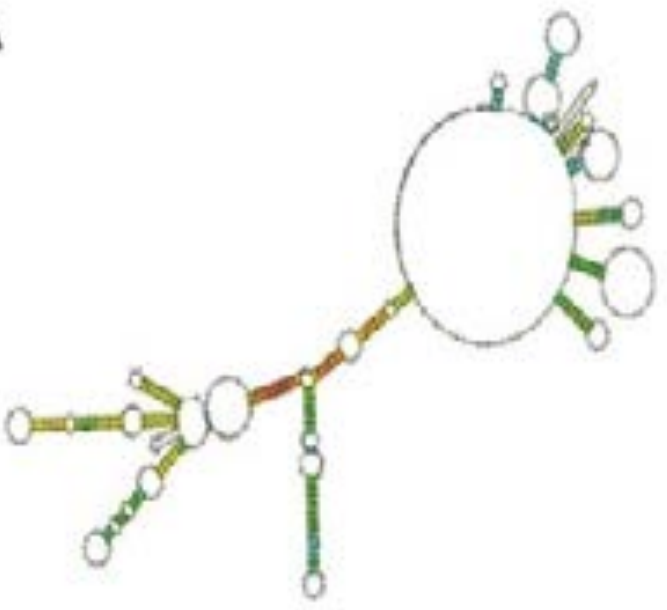

B

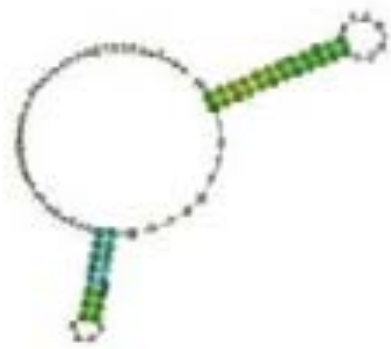




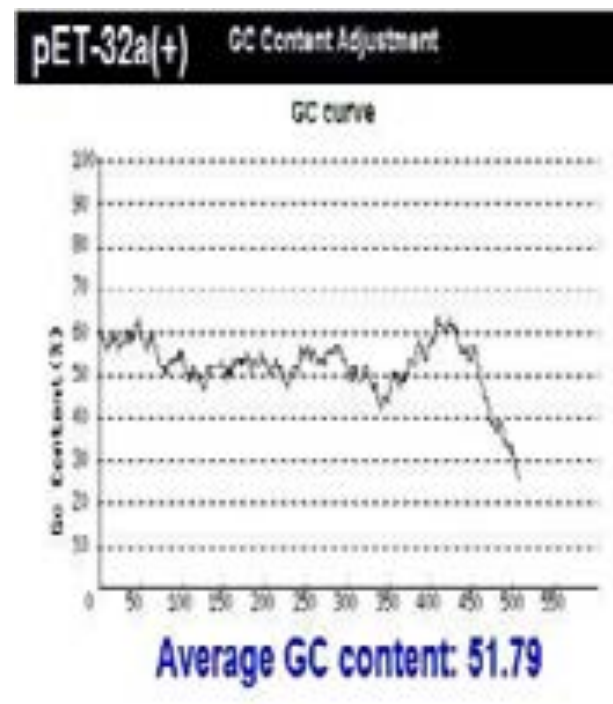

$\rightarrow$

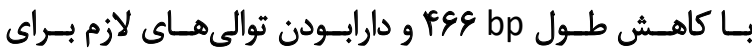

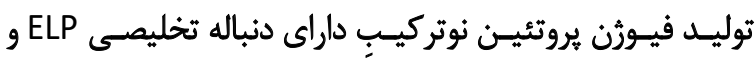

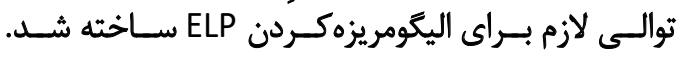

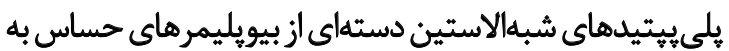

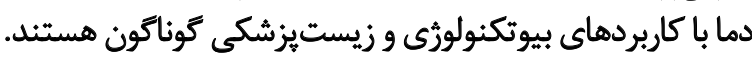

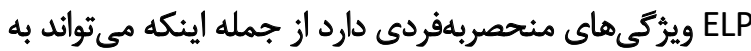

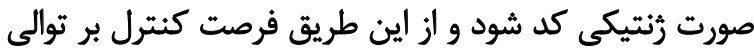

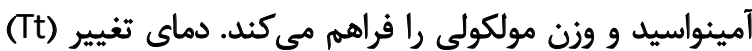

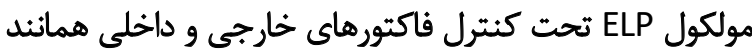

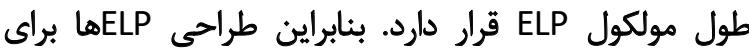

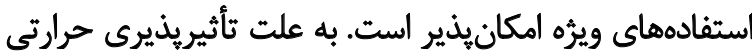

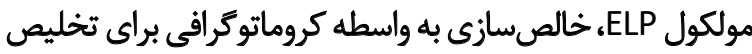

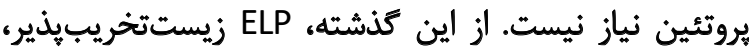

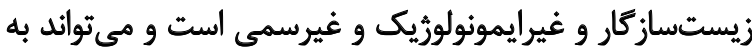

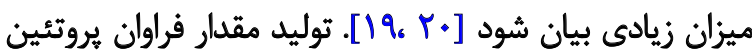

\section{DET-MOD GCConathositant}

GC curve

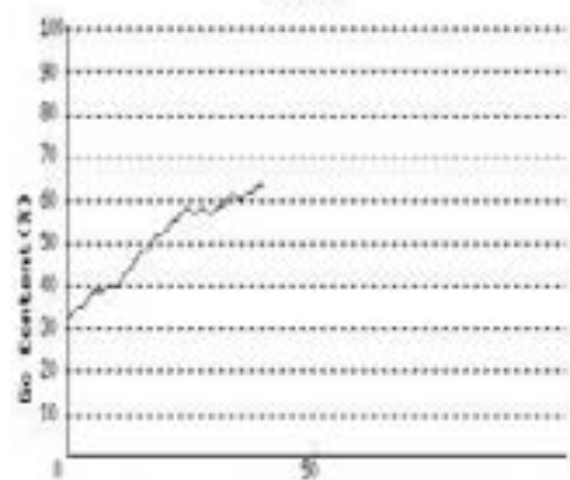

Average $G C$ content: $\$ 0.13$

Xbal Xhol دو X+C دو قطعه مابين دو سايت شناسايي Y. آناليز محتواي ؟). توالى هلاسميد pET-MOD حاصل با كد دردسترس است.

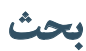

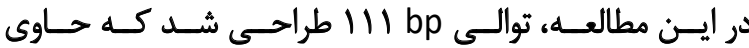

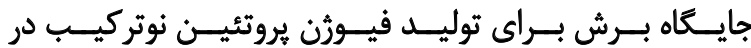

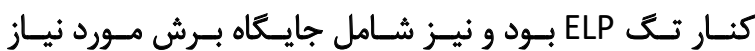

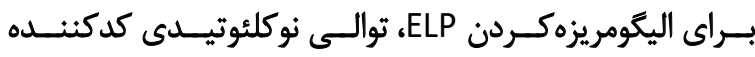
GGSGGSG

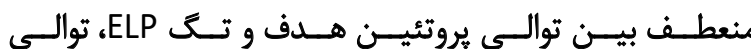

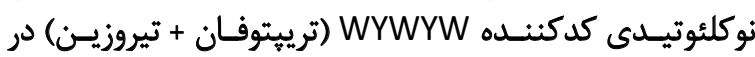

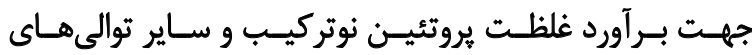

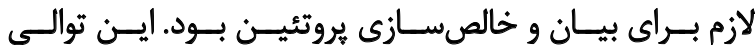

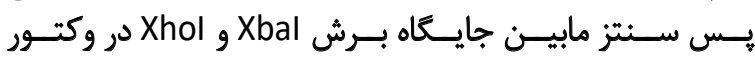

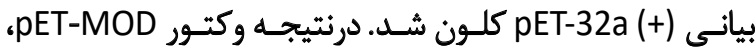

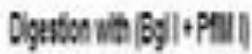

\section{GATCFTargat Protein) TTAA CCG.NPGXG).GGC}

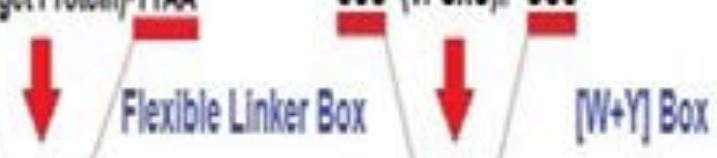

Xbal rbs BamHlEcool Sfil Xhol

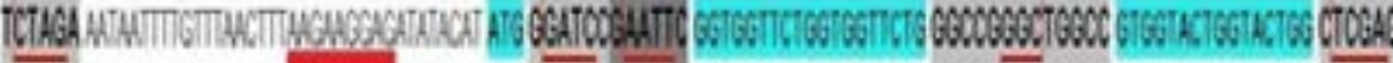
AGACI
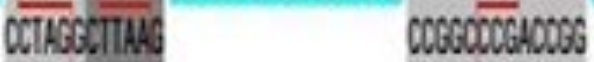

GACTC 


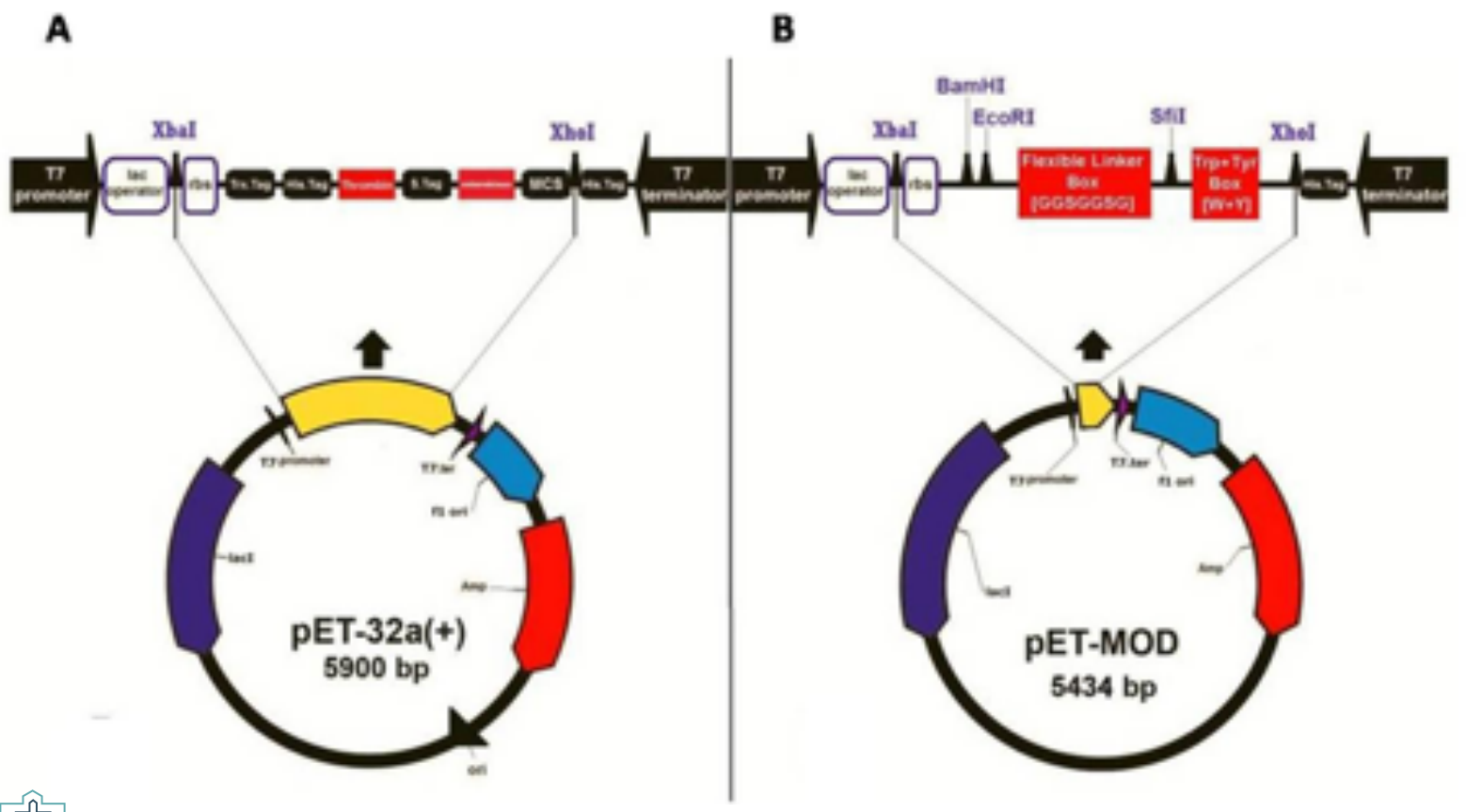

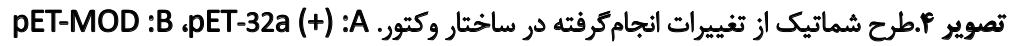

است بر اساس اندازهو جهت قراركيرى، همانئ بسيارى از تكها،

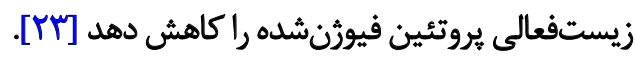

در مطالعات مختلفى كه در زمينه توليد فيوثن يروتئين

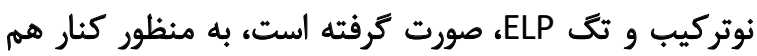

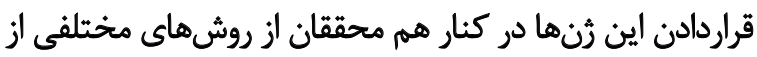

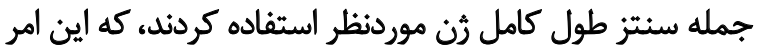

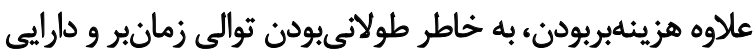

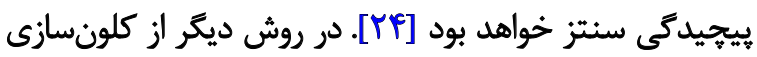

فيوزن زيستفعال، موضوع حياتى در بيوتكنولوزى است و ELP فيوزنه انتخاب مناسبى براى اين موضوع است. محل مريت مراركيرى

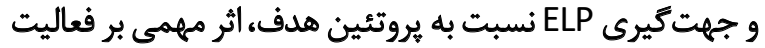

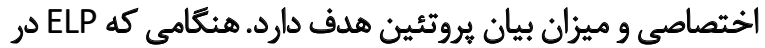

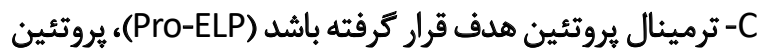

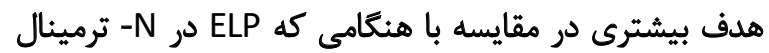

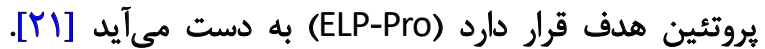

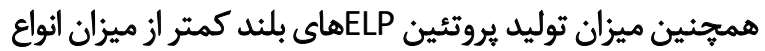

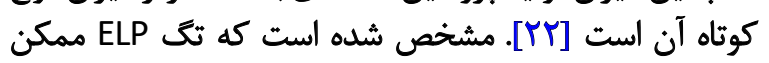

$\begin{array}{llllllll}1 & 2 & 3 & 4 & 5 & 6 & 7 & 8\end{array}$

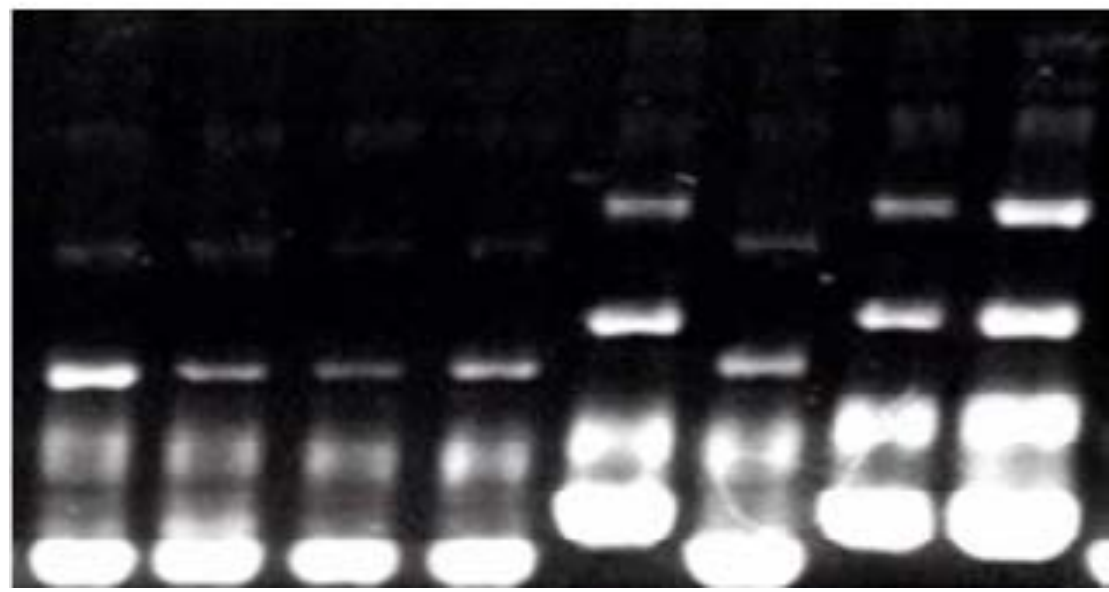

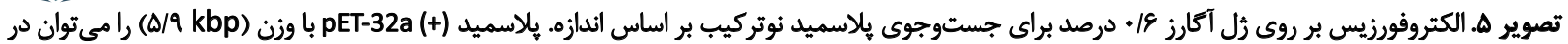

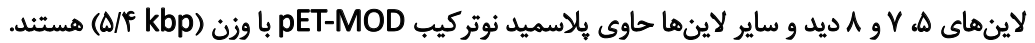




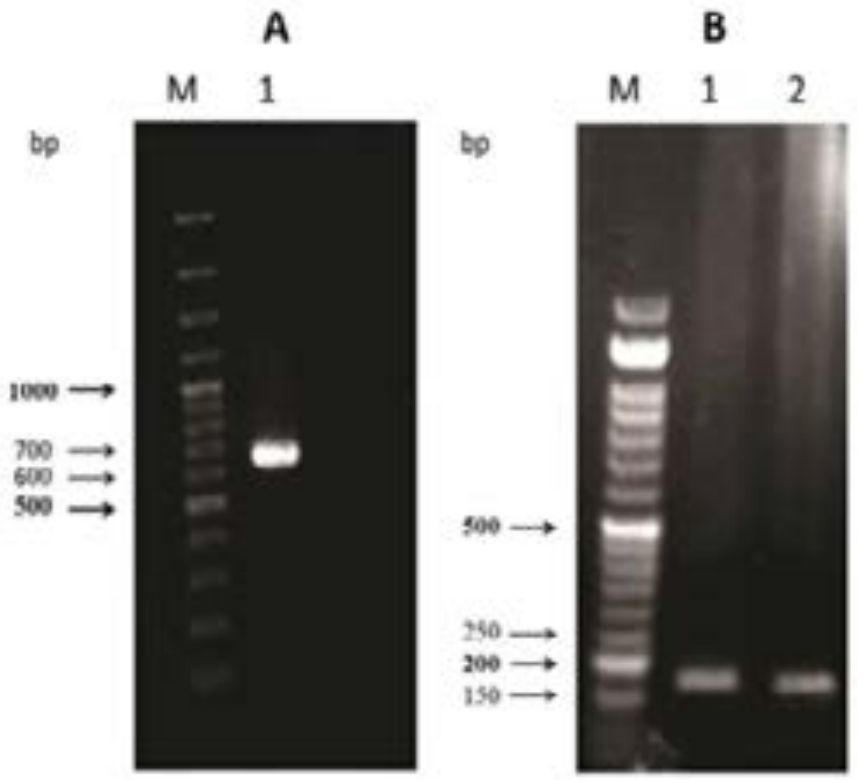

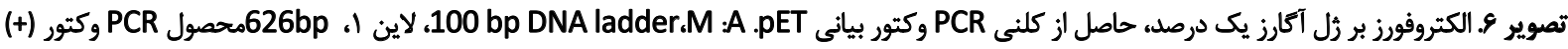

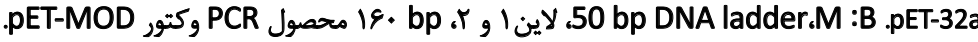

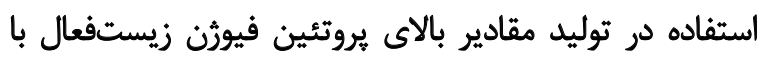

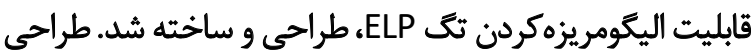

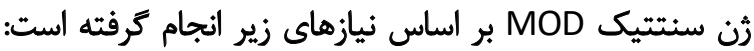

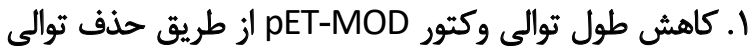

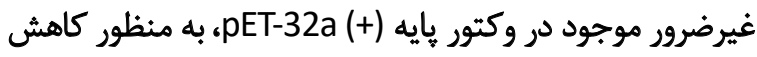

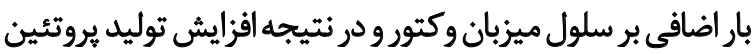

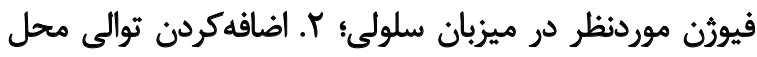

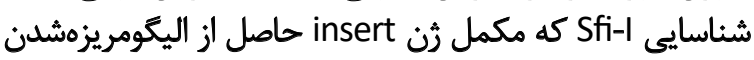

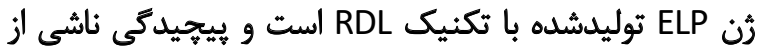

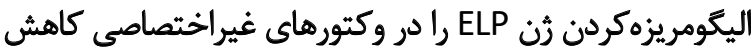

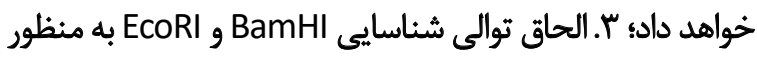

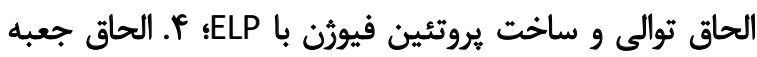

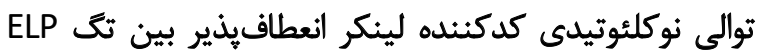

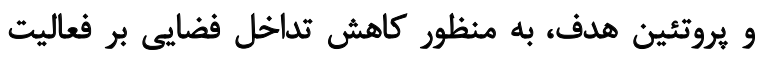

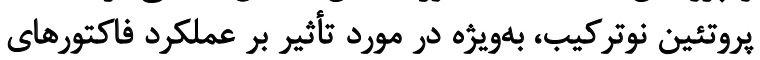

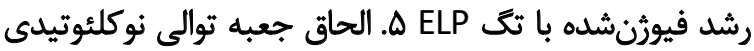

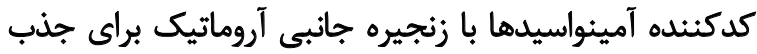

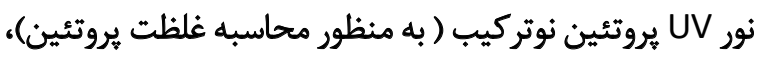

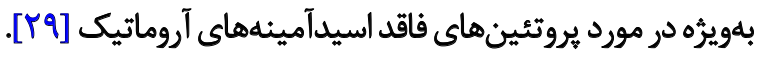
علاوه بر اين، pET-MOD داراى توالى 6XHis-Tag در انتهاى

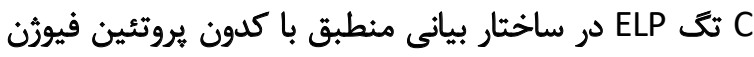

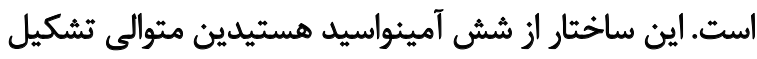

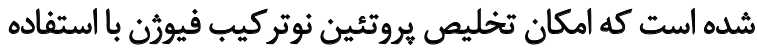

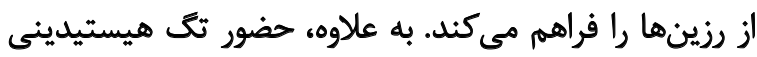

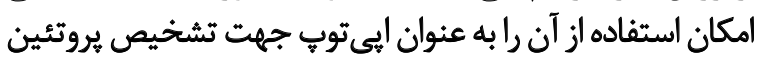

ابتدايى در وكتور غيربيانى و سيس ساب كلونينك استفاده شده

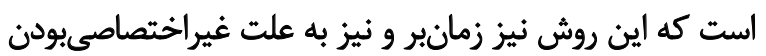

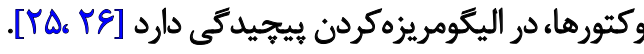
در مطالعه انجامشده توسط عمومى و همكاران، براى ساخت

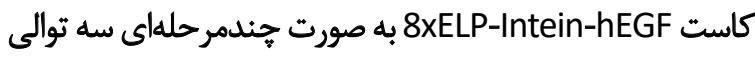

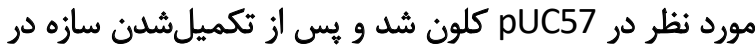

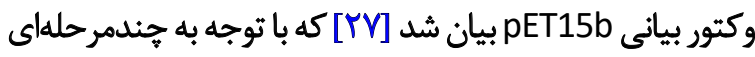

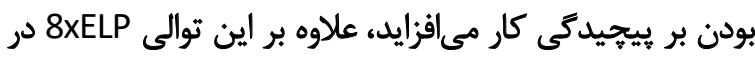

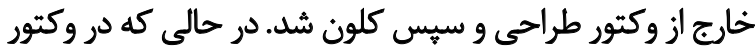

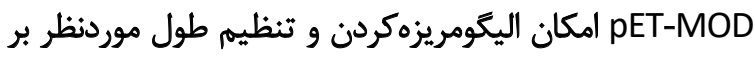

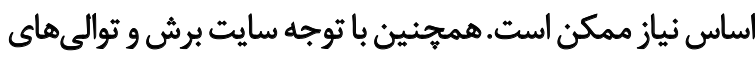

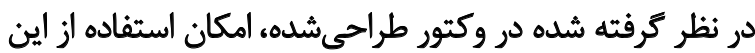

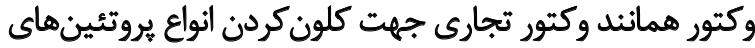

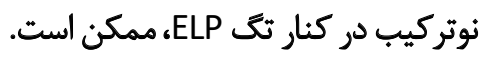
كروه تحقيقاتى ماء در مطالعات قبلى از وكتور دستكارى الشدها

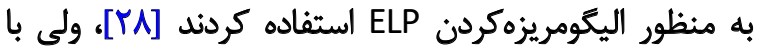

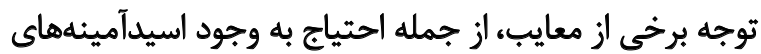

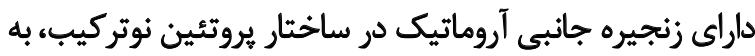

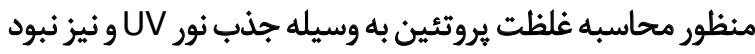

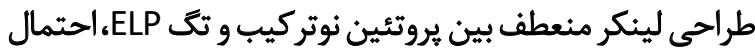

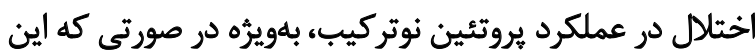

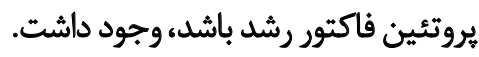

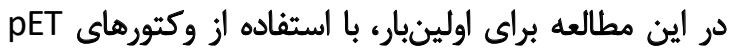

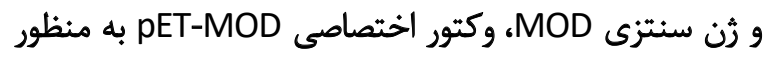


نويسندكان كمال قدردانى و امتنان را از معاونت يروهشى

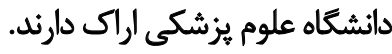

nitrilotri- با استفاده از آنتىبادى منوكلونال يا كونروكه با با آنزيم acetic acid-nickel

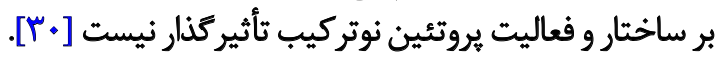

با باتوجه به امكان pET-MOD در خالصسازى يروتئين نوتركيب

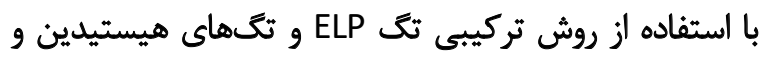

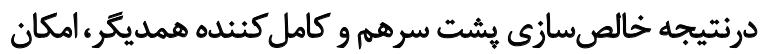

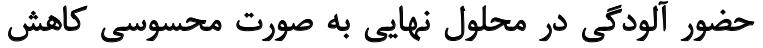

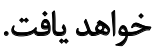

با توجه به ويزگكىهاى pET-MOD، استفاده از اين وكتور

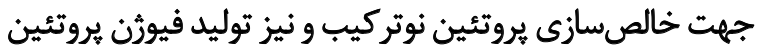

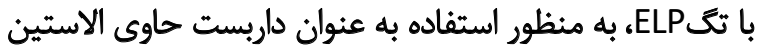

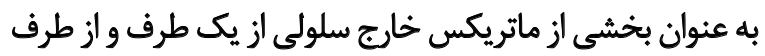

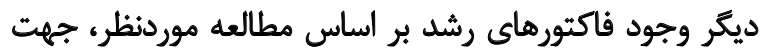

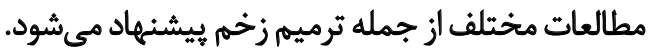

$$
\text { تثيجلئيرى }
$$

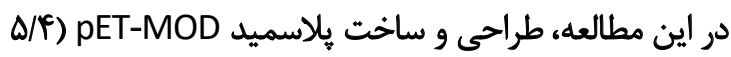

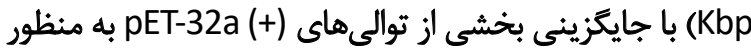

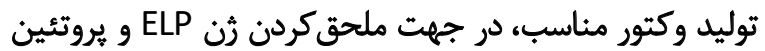
هدف و توليد بيومتريال فعال در مطالعات آتى انجام كرئ مرفت

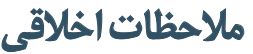

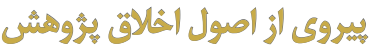

ايـن مطالعـه در كميتـهـ اخـلاق دانشـعاه علوميزشـكى اراك با

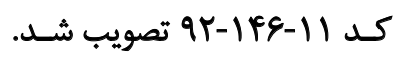

$$
\text { ماهي مالم }
$$

اين مقاله با حمايت مالى معاونت هُروهشى دانشكاه علوم

$$
\text { يزشكى اراك انجام شده است. }
$$

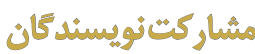

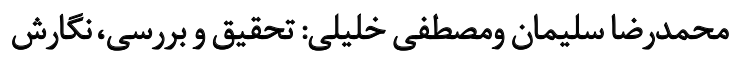

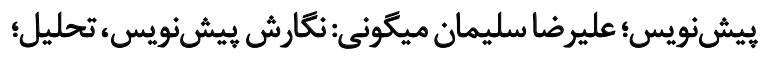

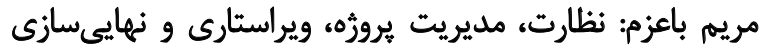

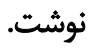

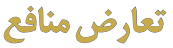

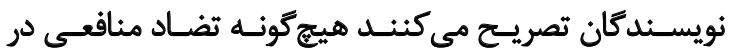

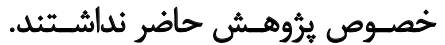




\section{References}

[1] Le DH, Sugawara-Narutaki A. Elastin-like polypeptides as building motifs toward designing functional nanobiomaterials. Mol Syst Des Eng. 2019; 4(3):545-65. [DOI:10.1039/C9ME00002J]

[2] Kouhi A, Yao Z, Zheng L, Li Z, Hu P, Epstein AL, et al. Generation of a monoclonal antibody to detect Elastin-Like Polypeptides. Biomacromolecules. 2019; 20(8):2942-52 [DOI:10.1021/acs. biomac.9b00503] [PMID]

[3] Duro-Castano A, Conejos-Sánchez L, Vicent MJ. Peptidebased polymer therapeutics. Polymers. 2014; 6(2):515-51. [DOI:10.3390/polym6020515]

[4] Coenen AMJ, Bernaerts KV, Harings JAW, Jockenhoevel S, Ghazanfari S. Elastic materials for tissue engineering applications: Natural, synthetic, and hybrid polymers. Acta Biomaterialia. 2018; 79:60-82. [DOI:10.1016/j.actbio.2018.08.027] [PMID]

[5] Meyer DE, Chilkoti A. Genetically encoded synthesis of proteinbased polymers with precisely specified molecular weight and sequence by recursive directional ligation: Examples from the elastin-like polypeptide system. Biomacromolecules, 2002; 3(2):357-67. [DOI:10.1021/bm015630n] [PMID]

[6] Fletcher EE, Yan D, Kosiba AA, Zhou Y, Shi H. Biotechnological applications of elastin-like polypeptides and the inverse transition cycle in the pharmaceutical industry. Protein Expr Purif. 2018; 153:114-20. [DOI:10.1016/j.pep.2018.09.006] [PMID]

[7] Kuna M, Mahdi F, Chade AR, Bidwell GL. Molecular size modulates pharmacokinetics, biodistribution, and renal deposition of the drug delivery biopolymer elastin-like polypeptide. Sci Report. 2018; 8(1):1-12. [DOI:10.1038/s41598-018-24897-9] [PMID] [PMCID]

[8] Floss DM, Schallau K, Rose-John S, Conrad U, Scheller J. Elastinlike polypeptides revolutionize recombinant protein expression and their biomedical application. Trends Biotechnol. 2010; 28(1):37-45. [DOI:10.1016/j.tibtech.2009.10.004] [PMID]

[9] Floss MD, Conrad U, Rose-John S, Scheller J. ELP-Fusion technology for biopharmaceuticals. Fusion protein technologies for biopharmaceuticals. In: Stefan R. Schmidt. Applications and Challenges. New Jersey: John Wiley \& Sons; 2013. [DOI:10.1002/9781118354599.ch14]

[10] McDaniel JR, Mackay JA, Quiroz FG, Chilkoti A. Recursive directional ligation by plasmid reconstruction allows rapid and seamless cloning of oligomeric genes. Biomacromolecules. 2010; 11(4):944-52. [DOI:10.1021/bm901387t] [PMID] [PMCID]

[11] Zhu Y, Hoshi R, Chen S, Yi J, Duan C, Galiano RD, et al. Sustained release of stromal cell derived factor-1 from an antioxidant thermoresponsive hydrogel enhances dermal wound healing in diabetes. J Control Release. 2016; 238:114-22. [DOI:10.1016/j.jconrel.2016.07.043] [PMID]

[12] Koria P, Yagi H, Kitagawa Y, Megeed Z, Nahmias Y, Robert Sheridan $R$, et al. Self-assembling elastin-like peptides growth factor chimeric nanoparticles for the treatment of chronic wounds. Proc Natl Acad Sci USA. 2011; 108(3):1034-9. [DOI:10.1073/ pnas.1009881108] [PMID] [PMCID]

[13] Ke C, Xiong H, Zhao C, Zhang Z, Zhao X, Rensing C, et al. Expression and purification of an ArsM-elastin-like polypeptide fusion and its enzymatic properties. Appl Microbiol Biotechnol. 2019; 103(6):2809-20. [DOI:10.1007/s00253-019-09638-w] [PMID]
[14] Akhani RC, Patel A, Patel MJ, Dedania S, Patel JS, Darshan H et al. Column chromatography free purification of recombinan $\alpha$-amylase from bacillus licheniformis by tagging with hydrophobic elastin like polypeptide. Biol Sci. 2018; 88(3): 1249-55 [DOI:10.1007/s40011-017-0862-z]

[15] Sarangthem V, Cho EA, Yi A, Kim SK, Lee BH, Park RW. Application of bld-1-embedded elastin-like polypeptides in tumor targeting. Sci Reports. 2018; 8(1):1-6. [DOI:10.1038/s41598-01821910-z] [PMID] [PMCID]

[16] Sambrook J, Fritsch E, Maniatis T. Molecular Cloning: A laboratory manual+ cold Spring Harbor. New York: Cold Spring Harbor Laboratory Press; 1989.

[17] Khalili M, Soleyman MR, Baazm M, Beyer C. High-level expression and purification of soluble bioactive recombinant human heparin-binding epidermal growth factor in Escherichia coli. Cell Biol Int. 2015; 39(7):858-64. [DOI:10.1002/cbin.10454] [PMID]

[18] Li W, Ng I, Fang B, Yu J, Zhang G. Codon optimization of 1, 3-propanediol oxidoreductase expression in Escherichia coli and enzymatic properties. Electron J Biotechnol. 2011; 14(4):7-17. [DOI:10.2225/vol14-issue4-fulltext-9]

[19] Hassouneh W, MacEwan SR, Chilkoti A. Fusions of elastin-like polypeptides to pharmaceutical proteins, in Methods in enzymology. Methods Enzymol. 2012; 502:215-37. [DOI:10.1016/B978-0 12-416039-2.00024-0] [PMID] [PMCID]

[20] Chilkoti A, Christensen T, MacKay JA. Stimulus responsive elastin biopolymers: Applications in medicine and biotechnology. Cur Opin chem biol. 2006; 10(6):652-57. [DOI:10.1016/j. cbpa.2006.10.010] [PMID] [PMCID]

[21] Conley AJ, Joensuu JJ, Jevnikar AM, Menassa R, Brandle JE. Optimization of elastin-like polypeptide fusions for expression and purification of recombinant proteins in plants. Biotechnol Bioengineer. 2009; 103(3):562-73. [DOI:10.1002/bit.22278] [PMID]

[22] Chu HS, Lee HK, Park JE, Kim DM, kim BG, Won Jl. Expression analysis of an Elastin-Like polypeptide (ELP) in a cell-free protein synthesis system. Enzyme Microb Technol. 2010; 46(2):87-91. [DOI:10.1016/j.enzmictec.2009.10.003]

[23] Shamji MF, Betre H, Kraus VB, Chen J, Chilkoti A, Pichika R, et al. Development and characterization of a fusion protein between thermally responsive elastin-like polypeptide and interleukin-1 receptor antagonist: Sustained release of a local antiinflammatory therapeutic. Arthrit Rheumat. 2007; 56(11): 3650-61. [DOI:10.1002/art.22952] [PMID]

[24] McPherson DT, Xu J, Urry DW. Product purification by reversible phase transition followingescherichia coli expression of genes encoding up to 251 repeats of the elastomeric pentapeptide GVGVP. Protein Expr Purif. 1996; 7(1):51-7. [DOI:10.1006/ prep.1996.0008] [PMID]

[25] Chen THH, Bae Y, Furgeson DY. Intelligent biosynthetic nanobiomaterials (IBNs) for hyperthermic gene delivery. Pharm Res. 2008; 25(3):683-91. [DOI:10.1007/s11095-007-9382-5] [PMID]

[26] Fujita $\mathrm{Y}$, Funabashi $\mathrm{H}$, Mie M, Kobatake E. Design of a ther mocontrollable protein complex. Bioconjugate Chem. 2007; 18(5):1619-24. [DOI:10.1021/bc070120x] [PMID]

[27] Omoumi N, Shokrgozar MA, Noormohammadi Z. Design and construction of recombinant ELP-Intein cassette for use in simple and new purification methods of recombinant proteins. Modern Med Lab J. 2017; 1(2):84-90. [DOI:10.30699/mmlj17.1.2.84] 
[28] Soleyman MR, Khalili M, Soleyman Meigoni A, Mohammadzadeh Ghasghaii H, Zendedel A, Baazm M. Gene cassette design, cloning and expression of recombinant elastin like polypeptide to produce a functional biomaterial in tissue engineering. Koomesh. 2016; 17(4):1006-16.

[29] Noble JE, Bailey MJ. Quantitation of protein, In: Noble JE, Bailey MJ.Methods in Enzymology. Amsterdam: Elsevier; 2009. [DOI:10.1016/S0076-6879(09)63008-1]

[30] Ramos CR, Abreu PA, Nascimento AL, Ho PL. A high-copy T7 Escherichia coli expression vector for the production of recombinant proteins with a minimal $\mathrm{N}$-terminal His-tagged fusion peptide. Braz J Med Biol Res. 2004; 37(8):1103-9. [DOI:10.1590/ S0100-879X2004000800001] [PMID] 